\title{
Handy Wheel Chair to help Disabled People Get into the Car
}

\author{
Tianqi Zhang ${ }^{1,2}$, Weilong $\mathrm{Xu}^{1,2}$, Shafiqul Md. Islam ${ }^{1,2}$, Xing $\mathrm{Wu}^{3}$ and Sharon Kao-Walter ${ }^{1,2,3, k}$ \\ ${ }^{1}$ Blekinge Institute of Technology, Dept. of Mech. Eng., SE 371 79, Karlskrona, Sweden, \\ ${ }^{2}$ Shanghai Second Polytechnic Univ., Faculty of Mech. \& El. Eng., 201209, Shanghai, China. \\ ${ }^{3}$ Kunming Institute of Science and technology, Faculty of Mech. \& El. Eng., 650500, Kunming, China.
}

\begin{abstract}
A wheelchair with a gear system was designed and analysed in order to find a good solution for people with wheelchair to move into the car. This gear system has been designed to be installed in the car and the whole wheelchair can be moved into the car automatically even with the people on it. The three dimensional design have been done in a CAD commercial program with this the solid mechanics analysis was also performed. Volvo XC 60 and $\mathrm{V} 60$ as well as a foldable wheelchair have been chosen in this work.
\end{abstract}

\section{Introduction}

Nowadays, cars are becoming more and more familiar to people. But still most of the cars do not friendly enough to the people with disability. If the disability people want to be the driver or passenger of car, they need to find a special car or get the help of other person. Therefore it is necessary to design a new system to help them to get into the normal family car automatically without an assistant. It will provide them with a new way to experience normal people's life. In fact, being able to take care of themselves use to be the biggest dream of the disabled people.

Several existed products have been found in the market [1-2]. Some of them need to get the help from an assistant and some of them need to have a big or special designed car. This work is a first step try to find a solution that the disability people can move into the normal family car by them self. The wheelchair in this study is mostly focused on a handy and foldable wheelchair which will be described in the following.

\section{Description of design solutions}

Many ideas have been discussed and one of them is selected based on the principle of interaction design which combined of the technological possibilities, user abilities and other criterion such as price, manufacturing and etc. In order to make the design size more close to reality, Volvo XC60 and V60 [3] as well as a foldable wheelchair have been chosen in this work. The design idea that has been chosen to work further consists two parts: A handy wheelchair (Figure 1) and a flexible guide rail structure need to be installed in the car. The flexible guide rail shall put up the whole wheelchair and move it into the car. This flexible guide rail is a gear system that controls the whole device to move up and down as well as rotate around.

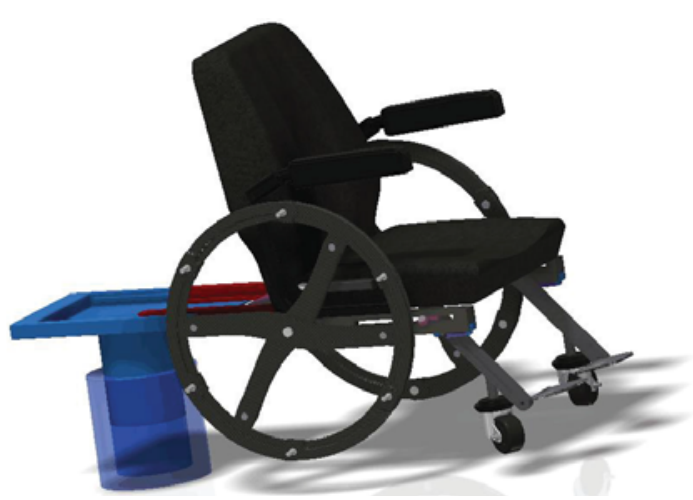

Figure 1. The wheelchair on the guide rail

\subsection{The operating steps of the handy wheelchair}

When the user want to move into the car for being passenger or even driver, they should perform the following steps which has also be illustrated in Figure 2: First of all, one should use the wheelchair to move to the close position of the car and open the door. Secondly, he/her needs keep a little distance from the car and use the controller of the electrical motor to control the flexible guide rail to stretch out. In third step, user should move the wheelchair to the certain position that the guide rail is under the wheelchair and then they can lock the guide rail and the wheelchair. The lock is installed in front of the seat of the wheelchair and between the user's two legs. In fourth step, one need use the remote control to make the guide rail to put up the wheelchair and pull up the front wheelchair. Finally, they use the remote control to move the whole wheelchair inside the car, rotate 90 degrees and close the door as illustrated in figure 2 where the real size car model is used to simulate the situation how to move the whole wheelchair into the car. 


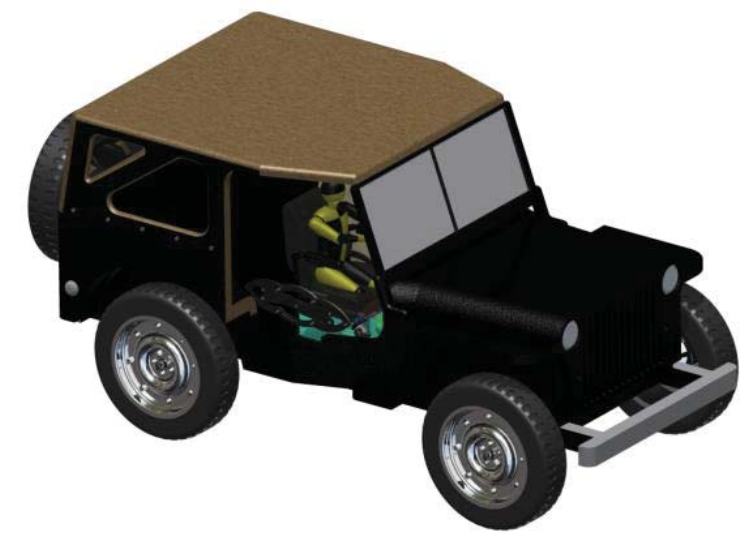

Figure 2. Wheelchair in the car and faced to the driving direction

Once the person reaches his destination, it is time to move outside of the car. The steps are as follow:

In the first step, the user should open the door with the remote control to rotate the guide rail 90 degrees and stretch outside the guide rails along with wheelchair. Secondly, user should restore the folded rear wheels into the normal shape (if the wheel is foldable) and push down the front wheels. In the third step, user needs to use remote control to make the guide rail to put down the wheelchair on the floor and unlock the wheelchair and guide rail. Using the remote control to restore the guide rail inside and closing the door as the final step.

Through the explanation of the operating steps of the handy wheelchair system, it can be found that all steps can be finished by the user himself/ herself. Besides, the strength of users should be of the normal level. Otherwise, it will be hard for them to fold the rear wheels and pull up the front wheel.

\subsection{The operating steps of the wheelchair lock}

The structure that locking the wheelchair on the guide rail is an important part to ensure the safety of the users. Two different parts are designed to protect the wheelchair from falling down from the guide rail. As shown in figure 3 , the user need to move the wheelchair towards the guide rail when the guide rail has been stretched out. After that, they will see the position of part B showing in the figure to ensure that they have been in the correct position. Next step is to make the user to use the remote controller to move up the guide rail to let the part $\mathrm{A}$ inside the groove of the guide rail board.

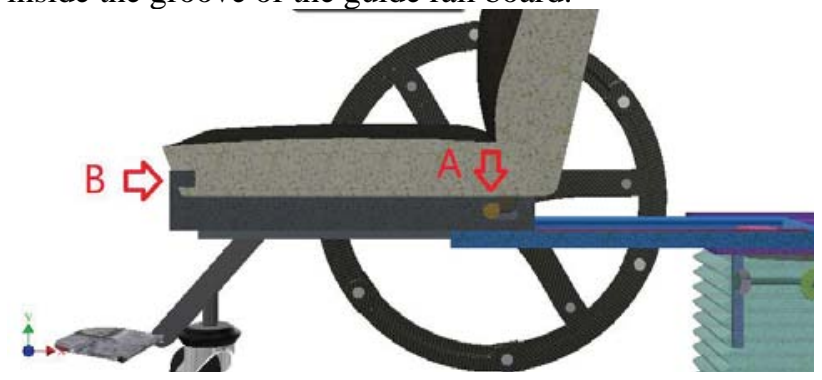

Figure 3. The status of the locked wheelchair to the guide rail
When the guide rail moves up, and make the part An inside the guide rail groove, the user of the wheelchair can move the wheelchair forward for a little distance to make the part B absolutely click into the wheelchair. At the same time, the part A will prevent from leaving the groove with the normal straight moving. Then the status of the wheelchair and the guide rail will become as shown in figure 3 . Finally, the gravity of the users and the force from other side will help the wheelchair to lock on the guide rail.

\subsection{The gear system}

A gear system is installed in the car, it is used to control the whole machine up and down, the guide rail out and in and the rotation of the wheelchair. The normal gear and two bevel gears are coaxial, and their rotary motion is controlled by electrical motor I (Figure 4). Besides, there is another electrical motor II to make rack do the straight movement, at the same time, the coaxial gears change their position. The electrical motors are powered by car battery, because the voltage is high enough and it is convenient to use.

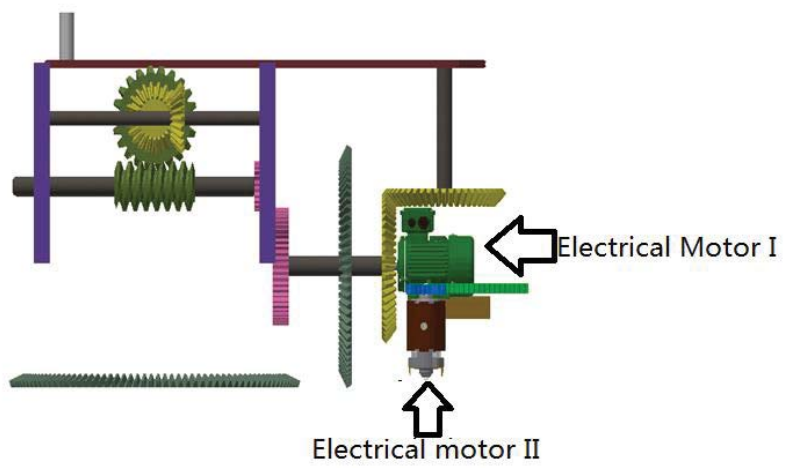

Figure 4. The two electrical motors and the gear system

The electrical control of the gear system have been designed by using the PLC technology. The details can be found in [4-5].

\section{The choice material}

The choice of the material of the designed system is an important point. It will influence whether the device is strength enough, the price and the weight. There are three requirements that:

First of all, gear system shall be strong enough and stable enough for carrying the wheelchair or a supporting device (see figure 5). Secondly, heavy weight will cause more trouble to wheelchair users, so it is necessary to reduce the mass of the whole wheelchair. At the same time, it is also needed to ensure that the wheelchair and gear system is strong enough. Finally, if it is turned into the reality, the price of the material is also a field which needs to be thought about. For example, some composite material will meet the first two requirements perfectly, but it may cost much more. This unnecessary expense will add in the sum cost of the product, the product will 
finally be a product with unreasonable price. Therefore, when the material is satisfied with first two requirements, the lower cost, the better. For example, Aluminium 6061 is the final material which is chosen for the main material of the wheelchair. Its features is found that aluminium 6061 has an ultimate tensile strength of at least $300 \mathrm{MPa}$ and yield strength of at least $241 \mathrm{MPa}$. Therefore, Aluminum 6061 will be chosen in the following Finite Element Method calculation as well as the hand calculation in order to make sure that the whole wheelchair system strong enough for daily use.

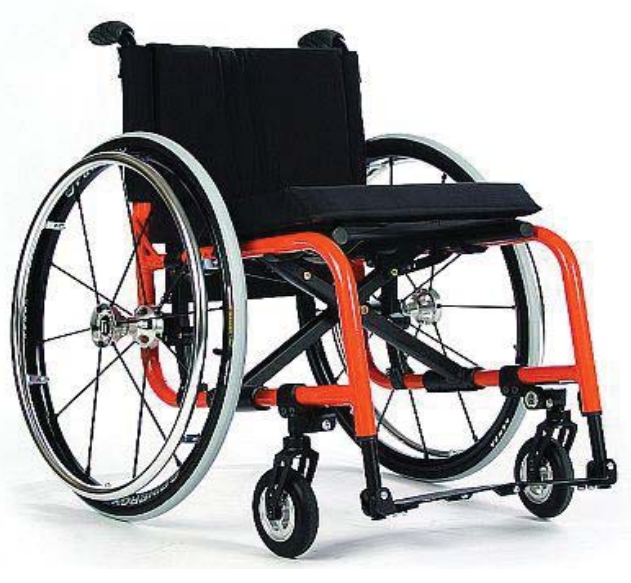

Figure 5. The wheelchair of Aluminium 6061 [6]

\section{Strength analysis of the structure}

A commercial CAD and Solid mechanics program Inventor 2015 [7] has been used to do the simulation with the applied force of $1000 \mathrm{~N}$ (as the weight of one male adult) on the chair of the wheelchair in order to check the strength of the guide rail. This strength calculation is to confirm that when the person move into the car with the help of guild gear, this designed guild gear will be strong enough to support the whole force even with the repeated loading case as shown in figure 6 .

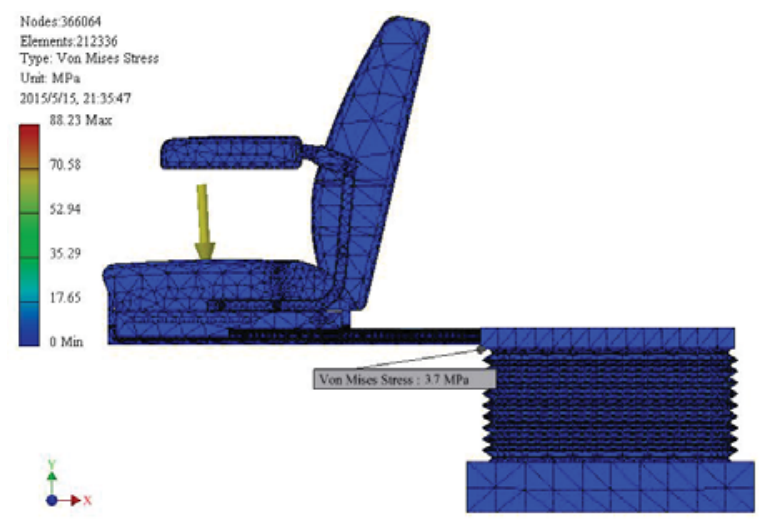

Figure 6. Stress analysis of the structure under repeated loading

Furthermore, the fillet weld that connect the hydraulic system with the car has also been calculated in order to find the right $a$ size.

\section{Conclusion and further work}

An idea to help the people with disability to move into a family or personal car has been verified. The entire model of a foldable wheelchair and a normal sized car have been used in this study. A typical gear system has been applied in this designed system which is installed in the car. The operating steps about how it works has been introduced and the strength of the system has also been analysed with the help of Finite Element Method. The assembling of the wheelchair with the gear system can be found in Appendix. Additionally, the electrical control is also added as one part of the whole wheelchair system. In the future work, the wheelchair can be optimized to be smaller, lighter and cheaper and the electrical control and the size of the wheelchair still has a large space to improve.

\section{References}

[1] "Nissan NV200 Skåp.", http://www.nissan.se/SE/sv/vehicles/lcv/NV200_VA N.html?cid=psmppTlGcHO_dc\#vehicles/lcv/NV200 VAN.

[2] "Professional solutions | Auto adapt.", http://www.autoadapt.com/en/pro/.

[3] "Volvo

XC60." http://www.volvocars.com/se/bilar/modeller/xc60.

[4] Xiaoli Wu, Junqing Liu, Yingya Huang, Design and Analysis of Man-machine System about Wheelchair (HoHai University, 2012).

[5] Tianqi Zhang, Weilong Xu, Bachelor Thesis, School of engineering, Department of Mechanical Engineering, Blekinge Institute of Technology, diva2:819460, 2015

[6] "TiLite Aero Z, now available in the folding Aero X".http://www.1stseniorcare.com/tiliteaeroznowavail ableinthefoldingaeroxtoofreeshippingintheus.aspx

[7] Inventor ${ }^{\circledR}$ Professional 3D CAD software, 2015

\section{Appendix}

\section{Assemble of the wheelchair}

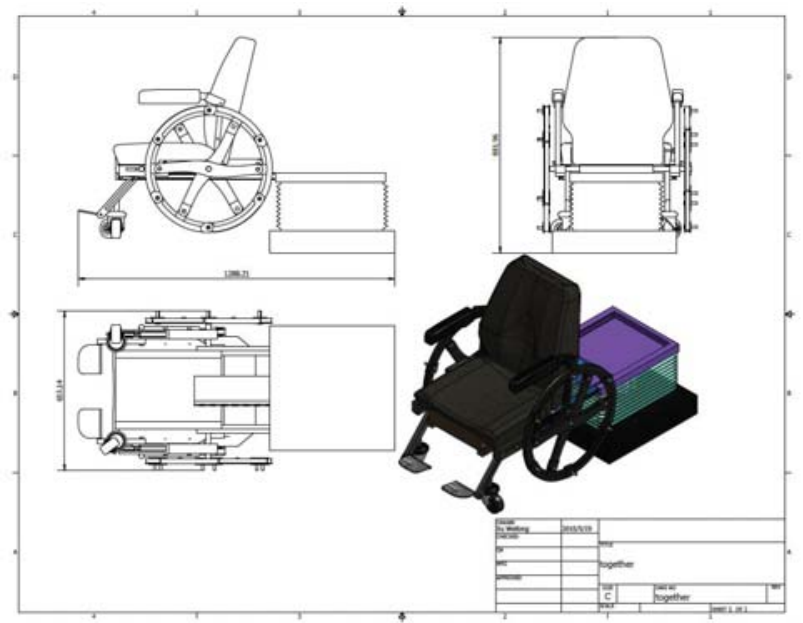

